\title{
Calculations of quantum oscillations in cuprate superconductors considering the pseudogap
}

\author{
E. V. L. de Mello ${ }^{1}$ \\ ${ }^{1}$ Instituto de Física, Universidade Federal Fluminense, 24210-346 Niterói, RJ, Brazi
}

\begin{abstract}
The observations of quantum oscillations frequencies in overdoped cuprates were in agreement with a charge density contained in a cylindrical Fermi surface but the measured frequencies of underdoped compounds were much smaller than expected. This was attributed to a topological transition into small pockets of Fermi surface associated with the existence of charge density waves. On the other hand, spectroscopic measurements suggested that the large two-dimensional Fermi surface changes continuously into a set of four disconnected arcs. Here we take into account the effect of the pseudogap that limits the available $k$-space area where the Landau levels are developed on the Luttinger theorem and obtain the correct total carrier densities. The calculations show how the disconnected arcs evolve into a closed Fermi surface reconciling the experiments.
\end{abstract}

The details of different Fermi surfaces in metals have long been explored by quantum oscillations (QO) experiments, like conventional de Haas-van Alphen effect. The quantum oscillations are a direct consequence of the quantization of closed Landau orbits perpendicular to an applied magnetic field. The energy of a given orbit depends on the applied field, and varying the field, there is always a resonance when a Landau level crosses the Fermi energy, from which the transverse (to the field) area of the Fermi surface is derived. The $\mathrm{QO}$ in the overdoped cuprate superconductor $\mathrm{Tl}_{2} \mathrm{Ba}_{2} \mathrm{CuO}_{2+\delta}$ (T12201) show the existence of a large Fermi surface covering nearly two-thirds of the Brillouin zone (BZ) ${ }^{1}$ in very good agreement with the angle-resolved photoemission spectroscopy (ARPES) results $\stackrel{2}{=}$. The frequency $(F)$ of oscillation is related with cross-sectional area $A_{\mathrm{k}}$ of the Fermi surface by the Onsager relation, $A_{\mathbf{k}}=2 \pi e F / \hbar$. Assuming that the Fermi surface is strictly two-dimensional, then the total itinerant carrier density per plane per area is given by Luttinger's theorem; $n=2 A_{\mathrm{k}} / A_{\mathrm{BZ}}=1+p$, where $A_{\mathrm{BZ}}=(2 \pi / a)^{2} \approx$ $265 \mathrm{~nm}^{-2}$ is the BZ area, $p$ is the average doping and $a$ the lattice parameter. These relations work quite well with several overdoped T12201 compounds $\mathrm{s}^{1-3}$ with $p \approx 0.25-0.30$.

However QO measurements in underdoped $\mathrm{YBa}_{2} \mathrm{Cu}_{3} \mathrm{O}_{y}$ (Y123) and in $\mathrm{HgBa}_{2} \mathrm{CuO}_{4+y}(\mathrm{Hg} 1201)$ are both 25-32 times lower than the overdoped T12201 frequency ${ }^{4-9}$, implying, by the Onsager relation, in very small cross-sectional areas $A_{\mathbf{k}}$. Consequently, there is a discrepancy with Luttinger theorem since typical doping differences are lower only by a factor of 2-3. To explain this overdoped/underdoped difference it was proposed a Fermi surface reconstruction into several small pockets of Fermi surface ${ }^{4-9}$. The change of sign in the Hall resistances with the temperature in high magneticfield-induced normal state of (Y123) and on Hg1201 suggested that these pockets are electron-like rather than holelike ${ }^{10,11}$. Since the negative Hall resistances occur between $p=0.07-0.15^{\frac{12}{2}}$, their existence and the crossover between electron-hole pockets were attributed to the incommensurate charge order $(\mathrm{CO})$ phase $\frac{10,13}{}$ or charge density waves $(\mathrm{CDW})$ superlattice formation ${ }^{14}$. Theoretical approaches beyond semiclassical approximations taking the effects of $\mathrm{CO}$ into consideration obtained signatures of electron pockets ${ }^{15}$ in qualitative agreement with the experimental data.

In order to check this Fermi surface reconstruction crossover an effort was made to perform angle resolved pho- toemission spectroscopy (ARPES) experiments with Y12316 and $\operatorname{Hg} 1201 \frac{17}{17}$, but they both did not detected the presence of electron pockets. Thus, the interpretation of $\mathrm{QO}$ in terms of electron and hole pockets differs markedly from singleparticle spectroscopy, suggesting that high magnetic fields might induce a new electronic state.

We provide a new interpretation to $\mathrm{QO}$ experiments taking into account the charge instabilities on Luttinger theorem, that was originally derived in the context of a Fermi liquid with uniform density. In previous papers 18 ? 19 we discussed the large amount of experimental evidences for spontaneous symmetry breaking and anomalous long-range ordered electronic states arising near the pseudogap (PG) temperature $T^{*}(p)$. The correlations between charge modulations wavelengths $Q_{\mathrm{CO}}\left(=1 / \lambda_{\mathrm{CO}}\right)$ in real space and the distance between the Fermi arcs tips in $k$-space that is dominated by the PG was established by scanning tunneling microscopy (STM) ${ }^{21,22}$ and by a combination of ARPES, STM and resonant $\mathrm{x}$-rays (REXS) on $\mathrm{Bi}_{2} \mathrm{Sr}_{2-x} \mathrm{La}_{\mathrm{x}} \mathrm{O}_{6+\delta}(\mathrm{Bi} 2201)^{23}$. On the theoretical side, we demonstrated that the PG energy $\Delta_{\mathrm{PG}}(p)$ is proportional to the ground state energy of a two-dimensional potential well with radius equal to $\lambda_{\mathrm{CO}}(p)^{\text {? }}$, establishing a direct connection between the CO spatial scale and the PG energy $\Delta_{\mathrm{PG}}(p)$. The charge anomalies are strong evidence of a nonFermi liquid behavior and pose the question of how does it affect the Luttinger theorem? We shall answer this question in the next paragraphs.

Another important property comes from measurements of the momentum or wave vector direction like Raman scattering ${ }^{24,25}$ and ARPES 26 that established the $d$-wave symmetry of the $\Delta_{\mathrm{PG}}(p)$. Because of their connection, we simulate the $\mathrm{CO}$ anomalies by a Cahn-Hilliard $(\mathrm{CH})$ approach in real space that is quite precise in reproducing the experimental observations $18,19,27,28$. In Fig. 11(a) we show an example of simulation; the $p=0.12$ compound from the Bi2212 family exhibiting $\mathrm{CO}$ with planar checkerboard structure in 100 vs. 100 unit cells similar to what we have done previously $\frac{19}{}$. Fig. 1 (b) is a plot made by Mathematica that displays constant energy cuts of the two-dimensional $k$-space single particle energy $\Delta_{\mathrm{PG}}(p, T)\left(k_{x}, k_{y}\right)=\Delta_{\mathrm{PG}}(p, T) \mid \cos \left(k_{x} a\right)-$ $\cos \left(k_{y} a\right) \mid / 2$. It vanishes near the (dark blue) diagonals or nodal directions and increases in the antinodal directions indicated by the white arrows pointing to larger energies represented by lighter colors. A simple two-dimensional inte- 


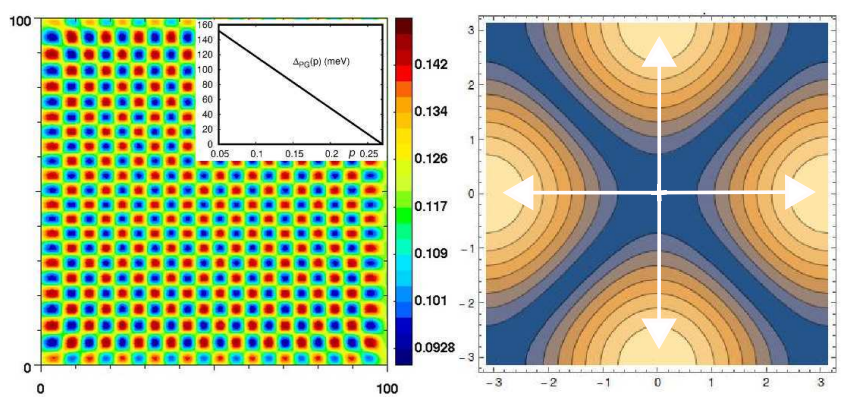

FIG. 1. The effect of the PG in real and $k$-space. (a) Checkerboard charge density map simulation for $p=0.12 \mathrm{Bi} 2212$ compound with hole-rich domains in red and hole-poor in blue. The inset shows the experimental linearly decreasing $\Delta_{\mathrm{PG}}(p, 0)$ energy amplitude in $\mathrm{meV}$ as function of $p^{24-26}$. In (b) we show the constant energy $d$ wave pseudogap $\left|\Delta_{\mathrm{PG}}\left(k_{x}, k_{y}\right)\right|$ color cuts which vanishes at the $\mathrm{BZ}$ diagonals $k_{x}= \pm k_{y}$, increases along the white arrows or nodal directions reaching the maxima at the antinodes. The dark blue region along the diagonals exemplifies a RBZ area (Eq. 1) since it is similar to the area inside the $p=0.10$ curves labeled "1" in Fig. 23 (a) and (b).

gral done also with Mathematica yields the area inside any these constant energy cuts. On the other hand, $\Delta_{\mathrm{PG}}(p, 0)$ decreases linearly with doping $24-26$ as shown in the inset of Fig. 11a) what implies generally in smaller $\Delta_{\mathrm{PG}}(p, T)$, that is, the constant energies curves are displace toward the antinodes Fig. 1 b) for overdoped materials. Consequently, the charges in underdoped compounds have larger energy constraints and their motion in $k$-space are limited to the nodal directions. In summary, the charge modulations in real space and energy restriction in momentum space are two manifestations of the PG effect in cuprates and we show below how to take into consideration in the Luttinger theorem. We have previously developed a theory that, at low temperatures, the two components SC order parameter (amplitude $\Delta_{\mathrm{sc}}$ and phase $\Phi$ ) is induced by lattice fluctuations in alternating $\mathrm{CDW}$ or $\mathrm{CO}$ domains 19,29 . The local SC phases are coupled by Josephson energy $\left.E_{\mathrm{J}}^{\mathrm{ij}}(T)=\left(\Delta_{\mathrm{sc}}\left(r_{\mathrm{i}}, T\right), \Delta_{\mathrm{sc}}\left(r_{\mathrm{j}}, T\right), R_{\mathrm{n}}\right), T\right)$, where $\Delta_{\mathrm{sc}}\left(r_{\mathrm{i}}, T\right)$ is the SC amplitude in the charge domain $i$ and $R_{\mathrm{n}}$ is the total resistance just above the SC transition like in granular ${ }^{19}$ ? or disordered superconductors ${ }^{30}$. The $E_{\mathrm{J}}^{\mathrm{ij}}(T)$ are the local superfluid phase stiffness $\frac{30}{} \rho_{\mathrm{sc}}^{\mathrm{ij}}$ and its average over the whole plane $\left\langle E_{\mathrm{J}}\right\rangle=\rho_{\mathrm{sc}}$ is directly proportional to the planar or two-dimensional superfluid density. In general, $n_{\mathrm{sc}}=\left(4 k_{\mathrm{B}} m^{*} / \hbar^{2}\right) \rho_{\mathrm{sc}}$ where $m^{*}$ is the effective mass of the electron ${ }^{31}$.

Now, under the extreme QO conditions; applied fields around $50 \mathrm{~T}$ and temperature $T \approx 1 \mathrm{~K}$, the local Cooper pairs or the SC amplitudes break down and the superfluid density $n_{\text {sc }}$ become low energy normal carriers, that is, $n_{\mathrm{sc}}=n$. As mentioned above, the superfluid phase stiffness $\rho_{\mathrm{sc}}$ gives this charge density and more, it determines also the energy scale or available kinetic energy of these unpaired holes. On the other hand, the PG or CDW charge instabilities are unaffected by a $50 \mathrm{~T}$ magnetic fields as demonstrated, for instance, by Changet al ${ }^{32}$. Consequently, in $\mathrm{QO}$ experiments the free car- riers from unpaired holes $\approx n$ are constrained by the $\mathrm{PG}$ to obey the following relation in the BZ,

$$
\rho_{\mathrm{sc}}(p, T=0 \mathrm{~K}) \geq\left|\Delta_{\mathrm{PG}}(p, 0)\left(k_{x}, k_{y}\right)\right| .
$$

This inequality defines the two-dimensional restricted $B Z$ area $(\mathrm{RBZ}(p))$ for particles under $\mathrm{QO}$ conditions and charge instabilities or in the presence of the PG, a restriction that does not exist for free particles. It means that the particles originally from the SC condensate are restricted to the region where the PG is weaker and, therefore it may not be energetically favorable to be near the anti-nodes $\left(k_{x}, k_{y}\right)=( \pm \pi / a, 0)$ or $(0, \pm \pi / a)$ regions. To illustrate, the RBZ for underdoped compounds that $\left|\Delta_{\mathrm{PG}}\right|$ is generally much larger than $\rho_{\mathrm{sc}}$ resembles the blue region near the BZ diagonals in Fig. 1(b). As $\left|\Delta_{\mathrm{PG}}\right|$ becomes small with increasing doping, the RBZ area becomes larger, eventually comprising the dark blue, the light blue, the dark brown and the lighter brown regions in Fig. 1 (b) and when $\left|\Delta_{\mathrm{PG}}\right| \rightarrow 0$ the RBZ becomes the full $A_{\mathrm{BZ}}=(2 \pi / a)^{2} \approx 265 \mathrm{~nm}^{-2} \mathrm{BZ}$ area.

To calculate explicitly the RBZ from Eq. (1), we obtain the $\rho_{\text {sc }}(T)$ values for Y123 and other cuprates from our previous paper? and $\Delta_{\mathrm{PG}}(p, 0)$ are taken from the experiments ${ }^{24,26}$. They are listed in Table I. We use Mathematica to plot the twodimensional curves $\rho_{\mathrm{sc}}(p, 0)=\left|\Delta_{\mathrm{PG}}(p, 0)\left(k_{x}, k_{y}\right)\right|$ for two Y123 underdoped compounds with $p=0.10$ labeled by " 1 ", 0.125 by " 2 " and one T12201 overdoped with $p=0.27$ by " 3 ", in Fig. 22 a) for electrons and Fig. 2 (b) for holes. We use also Mathematica to integrate directly the two-dimensional areas below these three curves the defines the RBZ. The areas or RBZ in the curves " 1 " and " 2 " in unit of $\mathrm{nm}^{-2}$ are listed in Table I.

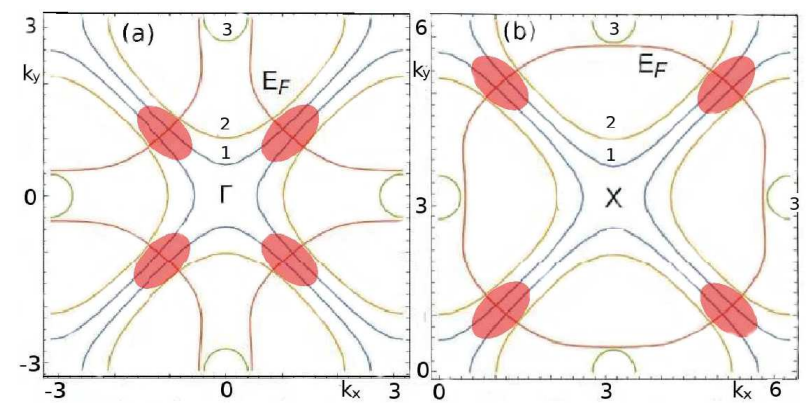

FIG. 2. Three curves $\rho_{\mathrm{sc}}(p, 0)=\left|\Delta_{\mathrm{PG}}(p, 0)\left(k_{x}, k_{y}\right)\right|$, for two Y123 underdoped compounds with $p=0.10$, labeled " 1 ", 0.125 "2" and a T12201 overdoped $p=0.27$ labeled " 3 " in the BZ for electrons (a) and in (b) for holes. The area inside the four branches of each curve, that is, from the lines toward the center $\Gamma$ in (a) or $\mathrm{X}$ in (b) defines the RBZ. We draw also the $E_{\mathrm{F}}$ for $p=0.27$ for comparison that is nearly the same for the other dopings. Under the QO conditions the free particles from underdoped superconductors cannot move around the $E_{\mathrm{F}}$ because the four PG branches act like strong potential barriers confining the carriers near the zone diagonals. We represent these constrained Landau levels schematically by the squeezed red circles or ellipsoids whose areas obey the Onsager relation.

In the far overdoped region $\Delta_{\mathrm{PG}}(p, 0) \rightarrow 0$ and, in this case, the particles from the SC condensate are not restricted 


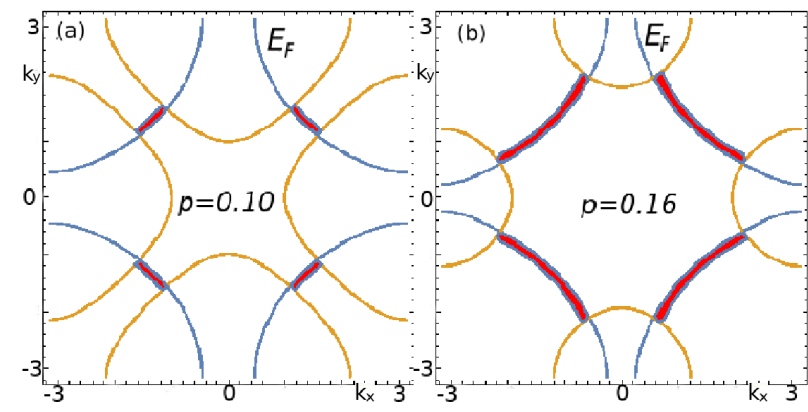

FIG. 3. The $d$-wave SC amplitude $\left|\Delta_{\text {sc }}\left(k_{x}, k_{y}\right)\right|$ at $T \approx T_{\mathrm{c}}+10$ $\mathrm{K}$. The same functional form of the PG and SC gap is an additional evidence of the connection between them. At these temperatures the size of the Fermi arcs or gapless regions are determined by thermal and quantum phase fluctuations, they increase and cover the whole Fermi surface when $T \rightarrow T^{*}$. To calculate the gapless arc sizes it is needed to take these two fluctuation effects into consideration like the derivations for three Bi2212 compounds in Fig. 6 of Ref. 19, after ARPES measurements ${ }^{36}$.

to the diagonals and can occupy the entire BZ. Therefore the Landau levels at the Fermi surface resembles the free particle case and go around describing almost perfect circles, like T12201 with $p=0.27$ shown by the red curve labeled by " 3 " in Fig. 2 (b), in total agreement with the experimental results $\stackrel{1}{1}$. For $p<0.27, \Delta_{\mathrm{PG}}(p, 0)$ increases as $p$ decreases and according the above discussion, the particles at the Fermi energy cannot penetrate in the antinodal region and are forced to stay near the BZ diagonals. In other words, the holes are confined to the nodal or diagonal directions as it is the case for $p=0.10$ and 0.125 . Under QO conditions of very high magnetic fields the particles can move only in small circles or ellipsoids around the four nodal portions or arcs of the Fermi surface as schematically displayed in Fig. 2 (a) and (b). We emphasize that we do not calculate the areas of these circles or ellipsoids, but their areas $A_{k}$ are obtained from the QO measured frequencies $F(p)^{4-9}$ and the Onsager's relation and listed in Table I.

Consequently, the measured $\mathrm{QO}$ frequencies $F$ on underdoped samples come from the holes oscillations around these four separated regions or Fermi arcs bound by $\left|\Delta_{\mathrm{PG}}(p, 0)\left(k_{x}, k_{y}\right)\right|$ shown in Fig. 2 a) and (b). The four contributions are alike and only one single frequency is measured experimentally yielding the area $A_{\mathbf{k}}(p)$ from the Onsager relation. Taking all the above into consideration, we rewrite Luttinger theorem as:

$$
n=1+p=A \times 2 A_{\mathbf{k}}(p) / \operatorname{RBZ}(p),
$$

where $\mathrm{A}=1$ for $p \geq 0.27$ and 4 otherwise to account for the four PG nodal regions that cross the Fermi level. As we have already explained, we calculate $\operatorname{RBZ}(p)$ with the zero temperature $\Delta_{\mathrm{PG}}(p, 0)^{24}$ and $\rho_{s c}(p, 0)$ from our previous paper? and from experiments ${ }^{34}$. The $\Delta_{\mathrm{PG}}(p, 0)$ and $\rho_{\mathrm{sc}}(p, 0)$ values used in the calculations for $p=0.09(\mathrm{Hg} 1201), 0.010,0.125$ (Y123) and $p=0.30$ (T12201) are all listed in Table I] We do not have data to calculate $\rho_{\mathrm{sc}}(0.09)$ for $\mathrm{Hg} 1201$ and we used that of Bi2212 in the preceding paper? because their similar
$T_{\mathrm{c}}(p)$ curves. It is known that $\rho_{\mathrm{sc}}(p, 0)$ is maximum near or further the optimum doping and is small in the underdoped region while $\Delta_{\mathrm{PG}}(p, 0)$ decreases linearly with $p$. Both effects combined imply that $\operatorname{RBZ}(p)$ decreases rapidly as $p \rightarrow 0$ and are much less than the full $\mathrm{BZ}$ area also listed in Table I. Using the areas $A_{\mathbf{k}}(p)$ derived from the $\mathrm{QO}$ measured frequencies $F$ by the Onsager relation and Eq. (2), we derive hole doping densities in very good agreement with the compounds used in the QO experiments $1-9$ as listed in Table I.

It is important to emphasize that the Landau levels at the Fermi surface constrained by the PG provides also an explanation to the existence of the four-hole pockets with the measured frequencies or areas. Our approach is also in qualitative agreement with the Fermi arcs measured by several ARPES experiments in the absence of a magnetic field $2,21,36-40$. We have previously demonstrated 19 that the Fermi arcs become finite at $T>T_{\mathrm{c}}$ due to thermal and quantum fluctuations of the local superconducting order parameter phase $\Phi\left(r_{i}\right)$. We have shown that the average superconducting amplitude $\left\langle\Delta_{\mathrm{sc}}\right\rangle$ is finite but the order parameter vanishes along the nodal direction due to average quantum and thermal phase fluctuations $\frac{19}{}$. In Fig. 3 we show the results of these phase fluctuation calculations for $p=0.10$ (a) and 0.16 (b) at $T \approx T_{\mathrm{c}}+10 \mathrm{~K}$ that reproduced ARPES measurements on Bi2212 $2^{36}$. We should mention that previous works connecting $\mathrm{QO}$ and $\mathrm{CO}$ anomalies that also reconciled QO and ARPES obtained only qualitative agreement with the experimental trends on underdoped compounds ${ }^{15}$ while we present detailed quantitative results for any doping level.

In summary, we demonstrated that taking the role of the PG in QO experiments we can understand why the measured frequencies $F$ that are generally proportional to the charge densities change by factor ten times larger than the average doping levels. We showed how low energy carriers, reminiscent from broken Cooper pairs under strong magnetic fields (the QO conditions) are constrained by the PG (or CDW instabilities) to the regions near the diagonals of the BZ. This new topological or geometric concept of a restricted BZ (RBZ) is the central idea of our work. This restriction in the BZ area modifies completely Luttinger's theorem that assumes the whole two dimensional $\mathrm{BZ}$ as the domain to the electrons on the $\mathrm{CuO}$ planes. The $d$-wave $\mathrm{PG}\left|\Delta_{\mathrm{PG}}\left(k_{x}, k_{y}\right)\right|$ plays the role of a two- dimensional non-constant potential in the BZ and the superfluid phase stiffness $\rho_{\mathrm{sf}}(p, 0)$ yields the energy scale to the carriers. We can think that the PG is the height of four symmetric mountains around a lake and $\rho_{\mathrm{sf}}$ is the level of the lake. We stress that this correction to the Luttinger's theorem is our main contributions to the understand the QO results and we do not calculate any frequency as function of magnetic field although strong fields are essential to transform the superfluid density in free particles. The RBZ in connection with the areas derived from the $\mathrm{QO}$ measured frequencies $F(p)$ through the Onsager's relations yields the correct hole doping $p$. The calculations provided clear quantitative results to the hole densities (listed in Table I), a new interpretation to the $\mathrm{QO}$ experiments and indicate a way to reconcile these observations with the Fermi arcs measurements by ARPES.

I acknowledge partial support by the Brazilian agencies 
TABLE I. We list the data and calculations of the four compounds used in QO experiments ${ }^{4-9}$. The measured frequencies in the third column are in tetrahertz $(\mathrm{T})$ and the respective areas from the Onsager relation are in the fourth column. $\rho_{\mathrm{sf}}(0)$ for Y123 is from the previous paper? ,

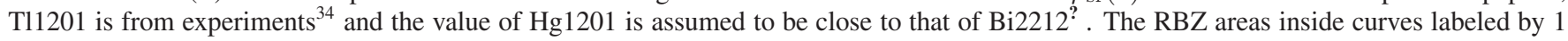
and 2 of Fig. 2 defined by Eq. 1 are calculated directly simply integrating with Mathematica the area inside these two plots. Notice that for $p=0.30$ in the last line the PG is zero the RBZ is equal the $\mathrm{BZ}$ area $=265 \mathrm{~nm}^{-2}$. The theoretical densities from Eq. 2 are in the last column and in agreement with the experimental values of column 1 .

\begin{tabular}{|c|c|c|c|c|c|c|c|}
\hline \hline$p$ & $T_{\mathrm{c}}$ & $F(p)(\mathrm{T})$ & $A_{\mathbf{k}}\left(\mathrm{nm}^{-2}\right)$ & $\Delta_{\mathrm{PG}}(\mathrm{meV})$ & $\rho_{\mathrm{sc}}(0)(\mathrm{K})$ & $\mathrm{RBZ} \mathrm{nm}^{-2}$ & $1+p(\mathrm{Eq}$. [2] \\
\hline $0.10(\mathrm{Y} 123)$ & $57 \mathrm{~K}$ & $530 \pm 20 \mathrm{~T} \mathrm{[4]}$ & 5.10 & $100.0 \mathrm{meV}[24]$ & 87.5 & $36.89(1)$ & 1.106 \\
\hline $0.125(\mathrm{Y} 123)$ & $64 \mathrm{~K}$ & $660 \pm 15 \mathrm{~T} \mathrm{[5}$ and 6$]$ & 6.37 & $91.0 \mathrm{meV}[24]$ & 92.5 & $45.26(2)$ & 1.126 \\
\hline $0.090(\mathrm{Hg} 1201)$ & $72 \mathrm{~K}$ & $840 \pm 30 \mathrm{~T} \mathrm{[8]}$ & 8.08 & $100.0 \mathrm{meV}[35]$ & 143.4 & 59.2 & 1.091 \\
\hline $0.30(\mathrm{~T} 12201)$ & $10 \mathrm{~K}$ & $18100 \pm 50 \mathrm{~T}[1]$ & 172.8 & $4 \mathrm{meV} \mathrm{[24]}$ & 29.5 & $265(3)$ & 1.303 \\
\hline
\end{tabular}

CNPq and FAPERJ.

* Corresponding author: evandro@ mail.if.uff.br

1 B. Vignolle et al., Nature 455, 952 (2008).

2 M. Platé et al., Phys. Rev. Lett. 95, 077001 (2005)

3 A. F. Bangura et al., Phys. Rev. B 82, 140501 (2010)

${ }^{4}$ N. Doiron-Leyraud et al., Nature 447, 565 (2007) 0801.1281

5 A. F. Bangura et al., Phys. Rev. Lett. 100, 047004 (2008)

${ }^{6}$ E. A. Yelland et al., Phys. Rev. Lett. 100, 047003 (2008)

7 C. Jaudet et al., Physical Review Letters 100, 187005 (2008)

8 N. Barišić et al., Nature Physics 9, 761 (2013)

9 M. K. Chan et al., Nature Communications 7, 12244 (2016)

10 D. LeBoeuf et al., Nature 450, 533 (2007)

11 N. Doiron-Leyraud et al., Physical Review X 3, 1 (2013)

12 S. Badoux et al., Nature 531, 210 (2016).

13 L. Taillefer, Journal of Physics: Condensed Matter 21, 164212 (2009)

14 S. Chakravarty, Science 319, 735 (2008)

15 A. Allais, D. Chowdhury, and S. Sachdev, Nature Communications 5, 5771 (2014)

16 M. A. Hossain et al., Nature Physics 4, 527 (2008)

17 I. M. Vishik et al., Physical Review B 89, 1 (2014)

18 E. V. L. de Mello, Europhys. Lett. 99, 37003 (2012)

19 E. V. L. de Mello and J. E. Sonier, Phys. Rev. B 95, 184520 (2017).

20 E. V. L. de Mello, J. Phys.: Cond. Matter 32, 40LT02 (2020)

${ }^{21}$ K. M. Shen et al., Science 307, 901 (2005)

22 W. D. Wise et al., Nature Physics 4, 696 (2008)
23 R. Comin et al., Science (New York, N.Y.) 343, 390 (2014)

24 S. Huefner, M. A. Hossain, A. Damascelli, and G. A. Sawatzky, Rep. Prog. Phys. 71, 062501 (2008)

25 N. Munnikes et al., Phys. Rev. B 84, 144523 (2011)

26 A. Damascelli, Z. Hussain, and Z.-X. Shen, Rev. Mod. Phys. 75, 473 (2003)

27 E. V. L. de Mello, R. B. Kasal, and C. A. C. Passos, J. Phys.: Condens. Matter 21, 235701 (2009)

28 E. V. L. de Mello and R. B. Kasal, Physica C: Superconductivity 472, 60 (2012)

29 E. V. L. de Mello and J. E. Sonier, J. Phys.: Condens. Matter 26, 492201 (2014)

30 B. I. Spivak and S. A. Kivelson, Phys. Rev. B 43, 3740 (1991)

31 I. Božović, X. He, J. Wu, and A. T. Bollinger, Nature 536, 309 (2016)

32 J. Chang et al., Nature Physics 8, 871 (2012)

33 E. V. L. de Mello, J. of Phys.: Cond. Matter 33, 145503 (2021)

${ }^{34}$ C. Niedermayer et al., Phys. Rev. Lett. 71, 1764 (1993)

35 Y. Li et al., Phys. Rev. Lett. 111, 187001 (2013)

36 W. S. Lee et al., Nature 450, 81 (2007)

37 M. R. Norman et al., Nature 392, 157 EP (1998)

38 T. Yoshida et al., Phys. Rev. B 74, 224510 (2006)

39 A. Kanigel et al., Nature Physics 2, 447 (2006)

40 T. Yoshida, M. Hashimoto, I. M. Vishik, Z.-X. Shen, and A. Fujimori, J. Phys. Soc. Japan 81, 011006 (2012) 